\title{
Modulation of laser-evoked pain perception and event-related potentials with non-invasive stimulation of the motor cortex
}

\author{
Ph.D. Thesis \\ Gábor Csifcsák, M.D.
}

Department of Psychiatry

Faculty of Medicine

Albert Szent-Györgyi Medical and Pharmaceutical Center

University of Szeged

Supervisor: Zoltán Janka, M.D., Ph.D., D.Sc.

External supervisor: Andrea Antal, M.Sc., Ph.D. 


\section{Summary}

In the last two decades new techniques of non-invasive brain stimulation have been introduced that enable relatively long-lasting and reversible facilitation or inhibition of distinct cortical areas by modulating the excitability of underlying neurons. Among these methods, repetitive transcranial magnetic stimulation (rTMS) and transcranial direct current stimulation (tDCS) are the most widespread ones. To date, both have been successfully used to modulate various perceptual, cognitive and motor functions in healthy subjects and several diseases, including chronic pain. Their efficacy regarding acute pain perception in healthy subjects however is still not well established.

The aims of our studies were to investigate the effects of a novel rTMS paradigm, continuous theta-burst stimulation (cTBS) and tDCS on laser-induced acute pain perception and laserevoked potentials (LEPs) when applied to the motor cortex of healthy adult volunteers. In two psychophysical and two electrophysiological experiments, we have compared the effects of real cTBS and two tDCS protocols (anodal and cathodal) to those of sham stimulations.

We have shown for the first time that cTBS over the motor cortex significantly alleviated laserinduced pain on both hands, accentuating on the contralateral limb. The effect of cTBS was accompanied by reduced N2-P2 LEP amplitudes in the case of medium intensity pain. In the tDCS experiments, cathodal stimulation of the motor cortex reduced mild pain contralateral to the side of stimulation. Moreover, cathodal tDCS attenuated N2-P2 LEP components, without modulating thresholds of medium intensity pain. On the contrary, anodal tDCS facilitated laser-induced warm sensation contralateral to the side of tDCS, without affecting either pain sensation or LEPs.

Our results indicate that non-invasive stimulation of the motor cortex causes antinociceptive effects that depend on the parameters of stimulation and are probably due to excitability changes in remote pain-related areas such as the operculoinsular region and the anterior cingulate cortex. These findings further strengthen the application of cTBS and tDCS in pain research, which might contribute to a more efficient manipulation of brain plasticity for therapeutic purposes. 


\section{Introduction}

Controlling pain has always been one of the biggest challenges of medical science. Despite pharmacological developments, still many patients suffer from long-lasting pain. During the last 40 years several surgical interventions have been used to modulate the activity of the central nervous system in order to control chronic, pharmacoresistant pain. Because such interventions may involve very serious adverse events, safer and at least equally efficient methods are still necessary. In the 90's new techniques of non-invasive brain stimulation have been introduced that enable the facilitation or inhibition of distinct cortical areas. Among these, transcranial magnetic stimulation (TMS) and transcranial direct current stimulation (tDCS) are the two most widespread ones.

\section{Transcranial magnetic stimulation}

TMS is based on electromagnetic induction, according to which a rapid, high-voltage alternating current is generated in a coil, producing a strong magnetic field that lasts for a few milliseconds. The magnetic field in turn induces electric currents in the cortex and stimulates the axons of neurons. When the stimulation is repeated with a certain frequency (repetitive TMS; rTMS), relatively long-lasting and reversible facilitatory or inhibitory effects can be induced in the stimulated area. rTMS has been successfully used to alleviate symptoms of various neurological and psychiatric diseases, including chronic pain. In pain syndromes the motor cortex is considered as one of the most effective target sites for stimulation.

A new stimulation protocol has been described recently, where the stimulation consists of three consecutive pulses of sub-threshold intensity that are presented rapidly (at 50Hz) and these bursts are repeated at the theta frequency $(5 \mathrm{~Hz})$. During continuous theta-burst stimulation (cTBS), this pattern lasts for 40 seconds, which causes robust inhibition for up to 60 minutes post-stimulation when applied over the motor cortex.

\section{Transcranial direct current stimulation}

tDCS is a neuromodulatory technique during which two electrodes connected to a stimulator are placed on the scalp. The intensity of the current is usually 1-2 $\mathrm{mA}$, which is flowing from the anode towards the cathode for maximally 20 minutes and causes shifts in the membrane 
potentials of neurons underneath the electrodes. Anodal stimulation depolarizes, while cathodal hyperpolarizes the stimulated area, hence exhibiting facilitatory and inhibitory effects respectively. Various tDCS protocols have been shown to efficiently modulate the excitability of motor, visual and somatosensory cortical areas. It has also been shown that anodal tDCS alleviates chronic pain when applied for 5 consecutive days over the motor cortex.

\section{The use of laser-evoked potentials for studying pain perception}

Electroencephalography (EEG) is a non-invasive method suitable for recording neural activity with excellent temporal resolution. When recorded during laser stimulation of the skin, laser evoked potentials (LEPs) can be analyzed at central and temporal scalp locations that are informative of the peripheral conduction of pain-evoked nerve impulses and their processing in distributed pain-related cortical areas. Based on their latencies, LEP waveforms can be divided into early (N1), late (N2 and P2) and ultra-late LEPs. The N1 typically appears around 160 milliseconds post-stimulation at bitemporal scalp sites. The late N2 and P2 potentials are maximal at the vertex, with latencies of 210 and 250 milliseconds in the case of hand stimulation. Generators of the N2 potential lie in operculoinsular cortices bilaterally and in the primary somatosensory cortex contralateral to the side of stimulation. Regarding the P2 potential, most source localizing studies reported it to be generated in the anterior cingulate cortex. The N2-P2 peak-to-peak amplitude correlates with the reported intensity of perceived pain, which is more likely due to modifications of the N2 amplitude. While early and late LEP components reflect A $\delta$-fiber-mediated neural responses, ultra-late LEPs appear 700-1300 milliseconds post-stimulation and are associated with C-fiber stimulation.

\section{Aims of the studies}

The aim of our first study was to investigate the effect of cTBS applied above the motor cortex on laser-induced acute pain perception and LEP parameters in healthy adult volunteers. Given the controversial antinociceptive effect of low and high frequency rTMS in A $\delta$-fiber mediated pain in healthy subjects, we chose the most effective TBS protocol (cTBS), which exhibits robust and relatively long-lasting inhibition of the motor cortex. We hypothesized that when compared to sham stimulation, cTBS would alter pain thresholds and LEP amplitudes on the hand contralateral to the side of motor cortex stimulation. For this purpose, we first carried out 
a psychophysical experiment (Experiment 1), where laser intensity was systematically increased and decreased. This enabled us to examine the efficacy of cTBS for three different laser intensities, evoking warm sensation, mild and moderate pain respectively. In the second experiment (Experiment 2), we aimed at inducing moderate pain in order to record LEPs before and after real and sham stimulation.

The second study was designed to assess the efficacy of anodal and cathodal tDCS of the motor cortex on laser-induced pain thresholds and LEP amplitudes. As for cTBS, we hypothesised that tDCS (primarily anodal stimulation) would significantly modulate pain thresholds, which would also be reflected in reductions of LEP amplitudes. We carried out a psychophysical experiment (Experiment 3) for assessing the effects of anodal and cathodal tDCS on warm sensation, mild, moderate and intensive pain and an EEG experiment (Experiment 4) during the course of which always moderate pain was induced to evoke reliable LEPs for analysis.

\section{Methods}

\section{Subjects}

Thirteen healthy right-handed volunteers ( 8 male and 5 female) between 18 and 30 years took part in Experiment 1, while 10 right-handed subjects ( 5 male and 5 female) aged between 2030 years participated in Experiment 2. Regarding the tDCS experiments, 16 (5 male and 11 female) subjects took part in Experiment 3, while 10 volunteers (5 male and 5 female) participated in Experiment 4. There was no overlap between the participants of the four experiments. All participants were informed about all aspects of the experiments and only those who signed an informed consent were included in the study. We conformed to the Declaration of Helsinki and the experimental protocol was approved by the Ethics Committee of the University of Göttingen. None of the participants suffered from chronic pain syndromes nor took any medication regularly. All participants participated in the two cTBS (real and sham stimulation) or in the three tDCS (sham, anodal and cathodal stimulation) sessions within the experiments that were separated by at least 5 days in order to avoid the effect of interference. The order of the real and sham sessions was counterbalanced across subjects. 


\section{Laser stimulation and pain evaluation}

Pain was elicited using a Tm:YAG laser system (WaveLight Laser Technologie AG, Erlangen, Germany). The thulium laser emits near-infrared radiation and allows the emitted heat energy to be precisely restricted to the termination area of primary nociceptive afferents without affecting the subcutaneous tissue. The distal handpiece of the laser was positioned $30 \mathrm{~cm}$ from the radial part of the dorsal surface of the hand. In both experiments the right hand was stimulated first in half of the cases; in the other half we started with the left hand.

We used a numeric analogue score (NAS) to assess the subjective intensity of pain. We instructed the subjects to pay attention to the laser stimuli and to rate the perceived pain verbally with numbers ( 1 for warm, between 1.1 and 1.9 from mildest to most intensive pain) about 2-3 seconds after each stimulation. Inter-stimulus interval varied between 8 and 15 seconds.

In the psychophysical experiments (Experiments 1 and 3) we applied two series of stimuli for each hand before (cTBS and tDCS), immediately after (cTBS and tDCS) and 30 minutes after stimulation (cTBS). We systematically increased laser intensity from $200 \mathrm{~mJ}$ in $50 \mathrm{~mJ}$ steps until subjects reported moderate pain. Then the laser energy was decreased from that intensity again in $50 \mathrm{~mJ}$ steps. This stimulation protocol was repeated twice. Hence we obtained four pain rating scores for each laser intensity prior to and following cTBS or tDCS for each hand and simulation type.

In the electrophysiological experiments (Experiments 2 and 4) in order to get reliable LEP waveforms, we aimed to induce medium intensity pain. We started with a laser intensity of 1.5-1.6 times of the threshold level during EEG recording and adjusted laser energy manually in order to keep the magnitude of pain between NAS scores 1.4-1.6. We delivered 40 laser pulses to each hand before and after cTBS and tDCS.

\section{Theta-burst stimulation}

In Experiments 1 and 2, real and sham cTBS was applied over the hand area of the left motor cortex. In Experiment 1, we used a standard, figure-of-eight-coil (MC-B70 Butterfly Coil, Magventure A/S, Farum, Denmark) and MagPro stimulator (Medtronic, Denmark) with an outer half-coil radius of $75 \mathrm{~mm}$, while a figure-of-eight-coil with an outer half-coil radius of 
90 mm (Dantec S.A., Skovlunde, Denmark) and a Magstim Super Rapid stimulator (Magstim Company Ltd., Whitland, Wales, UK) were used in Experiment 2. Stimulus intensity was $80 \%$ of the active motor threshold. The pattern of cTBS consisted of bursts containing 3 pulses at $50 \mathrm{~Hz}$ which were repeated at $200 \mathrm{~ms}$ intervals (i.e. $5 \mathrm{~Hz}$ ) for 40 seconds (resulting 600 pulses in total) continuously. In accordance with earlier studies, we stimulated above the position of electrode $\mathrm{C} 3$, which is situated above the primary motor cortex. In a separate experimental session, sham stimulation was applied with the same cTBS protocol using a sham coil (MC-PB70, Magventure A/S, Farum, Denmark) held at the same position. The participants were blinded concerning the type of the magnetic stimulation (real or sham).

\section{Direct current stimulation}

In Experiments 3 and 4, we applied anodal, cathodal and sham tDCS by a battery driven constant current stimulator (NeuroConn, Ilmenau, Germany) using a pair of rubber electrodes placed in a $5 \times 7 \mathrm{~cm}$ synthetic water-soaked sponge. One electrode was placed at position $\mathrm{C} 3$, while the other was situated above the right eyebrow. The type of stimulation (anodal or cathodal) refers to the polarity of the electrode above motor cortex. The current was applied for 10 minutes with an intensity of $1.0 \mathrm{~mA}$, while for sham stimulation it was turned on only for a few seconds to provide the slightly itchy sensation at the beginning of the stimulation. Subjects were not aware of the polarity and type of tDCS.

\section{Electroencephalographic recording}

In Experiment 2, EEG was recorded using a 64-channel montage (EasyCap; Falk and Minow Gmbh, Munich, Germany), whereas in Experiment 4, we could only use a five channel montage (Fz, Cz, Pz, T3 and T4) to enable the placement of the large tDCS electrodes. EEG electrodes were placed in accordance with the extended international 10-20 system. Data were always collected with the BrainAmp system (Brain Products GmbH, Munich, Germany) and were analysed off-line. In Experiment 2, the N2-P2 components were analyzed at three scalp regions: central (FCz, Cz, Pz), left (FC3, FC5, C3, C5, CP3, CP5) and right (FC4, FC6, C4, C6, CP4, CP6). In Experiment 4, we analysed baseline N1 amplitudes on channels T3 and T4, while N2 and P2 amplitudes were measured on all five channels. 


\section{Data analysis}

In Experiment 1, NAS values were divided into three groups according to the intensity of the laser stimulation, resulting in low intensity 200-399 mJ, medium intensity 400-599 mJ and high intensity 600-800 mJ groups. A repeated measures analysis of variance (ANOVA) was calculated with the mean NAS values across participants and was entered as dependent variables, while hand (left or right), condition (sham vs. cTBS), time (before, after and 30 minutes after) and intensity of laser stimulation (low, medium and high) served as independent variables. For post-hoc comparison Tukey's HSD test was used.

In Experiment 2, we normalized the data by dividing pain rating scores (1-9) by the actual laser energy $(\mathrm{J})$. These values were compared for both hands separately with repeatedmeasures ANOVA, where condition (real and sham cTBS) and time (before and after cTBS) served as within-subject factors. N2 and P2 baseline amplitudes and the N2-P2 peak-to-peak amplitude were entered into repeated-measures ANOVA for both hands separately.

In Experiment 3, we grouped the obtained pain rating scores into four perceptual categories: warm sensation (NAS =1), mild (NAS between 1.1-1.3), medium (NAS between 1.4-1.6) and intensive pain (NAS between 1.7-1.9). NAS values for each hand and pain category were compared between the three tDCS conditions with a 2-way ANOVA.

In Experiment 4, laser energies necessary to induce moderate pain were compared with repeated-measures ANOVA for both hands, where condition (anodal, cathodal and sham tDCS) and time (before and after tDCS) were entered as within-subject factors. Regarding LEPs, N1, N2, and P2 baseline amplitudes were entered into repeated-measures ANOVA for both hands separately. Student's $t$-test was used to compare the baseline amplitudes between different conditions. 


\section{Results}

cTBS experiments

In Experiment 1, low intensity laser stimulation (200-399 mJ) was either unperceivable or caused only a warm sensation in most of the participants. In the case of medium intensity laser stimulation (400-599 mJ) mean NAS values indicated pain sensation around threshold. Highintensity laser stimulation $(600-800 \mathrm{~mJ})$ resulted in a moderate-to-intensive pain sensation in all participants. Regarding right hand laser stimulation, there was a significant effect of time in the medium intensity group if real cTBS was applied (a reduction in NAS values before vs. immediately after and before vs. 30 minutes after cTBS). In the high intensity group there was a significant decrease in NAS only immediately after cTBS. We found a significance difference between conditions (cTBS vs. sham) only in the medium-intensity group. All of the other comparisons were not significant. In case of left hand stimulation there was a significant effect of time only in the high intensity group 30 minutes after cTBS. Comparing the two conditions (cTBS vs. sham) there were significant differences 30 minutes post-stimulation in the low-intensity group and in the medium-intensity group.

In Experiment 2, the N2 amplitude decreased in both cTBS conditions for both hands, but we found a significant time $\mathrm{x}$ condition interaction only in the case of right hand laser stimulation, indicating that after real cTBS the N2 amplitude reduction was larger than after sham stimulation. With regard to the P2 amplitude, we found a significant time $\mathrm{x}$ condition interaction only for left-hand laser stimulation, where the magnitude of amplitude change was significantly less when real cTBS was used.

\section{tDCS experiments}

In Experiment 3, we found a significantly reduced warm threshold for the right hand after anodal tDCS, when compared to the effects of sham stimulation. On the contrary, cathodal stimulation significantly increased laser intensities that were needed to induce mild pain when compared with sham or anodal stimulation. Neither medium nor high intensity pain thresholds were modulated by real tDCS. 
In Experiment 4, we did not find significant difference among the three tDCS conditions with regard to the laser intensities necessary to induce moderate pain. In case of the N1 component there was no significant time $\mathrm{x}$ condition interaction for either hands. In the case of the N2 component however, we found a significant time $\mathrm{x}$ condition interaction only when the right hand was stimulated. When compared with sham and anodal tDCS, cathodal stimulation significantly diminished the $\mathrm{N} 2$ amplitude. In contrast to cathodal stimulation, anodal stimulation did not affect the N2 amplitude when compared with sham tDCS. With regard to the P2 amplitude, the repeated-measures ANOVA revealed a significant modulatory effect of tDCS in the case of the right hand. The interaction of time and condition was significant for the cathodal-sham, marked but not significant for the anodal-sham and not significant for the anodal-cathodal comparison.

\section{Discussion}

In Experiments 1 and 2, we have shown that cTBS applied above the motor cortex alleviates experimentally induced acute pain and corresponding LEP amplitudes. In Experiment 1, the reduction of the pain perception was dominantly contralateral to the side of the cTBS (for medium and high intensity laser stimulation) and was observed immediately post-stimulation for medium-level laser intensity (around pain threshold). Furthermore, it remained stable for up to $30 \mathrm{~min}$ after stimulation. In case of the left hand, the significant effect of the cTBS appeared to be delayed. In Experiment 2 we have also shown that parallel to the contralateral-sided reduction of moderate intensity pain sensation after cTBS, real stimulation reduces the N2-P2 LEP complex, which is primarily due to smaller N2 amplitudes.

The fact that cTBS over the motor cortex attenuated the N2-P2 complex points towards the fact that remote effects should also be considered, since these LEP components reflect the activities of the bilateral operculoinsular and anterior cingulate cortices. Given that the motor cortex is intensively interconnected with the anterior cingulate cortex and that invasive stimulation of the primary motor cortex induced regional blood flow changes in several pain-related structures, it seems plausible that cTBS in Experiments 1 and 2 caused similar changes in these regions, which manifested in reduced LEP components. 
In Experiment 3, cathodal tDCS over the motor cortex resulted in diminished perception of mild pain, while anodal tDCS facilitated warm sensation. All these effects were only present when the hand contralateral to the side of tDCS was stimulated with the Tm:YAG laser.

Regarding LEP components in Experiment 4, we found significantly decreased N2 and P2 amplitudes after cathodal tDCS on the contralateral hand, while subjective pain ratings were not altered. One possible explanation for the observed effects is that the diminution of both LEP components after cathodal tDCS reflects a modulation of neural excitability in the primary somatosensory cortex, the operculoinsular region and/or the anterior cingulate cortex. Indeed, in a PET study, changes in regional cerebral blood flow in the right cingulate cortex and the right thalamus were reported while the investigators used exactly the same stimulation protocol for modifying motor cortex excitability as we did in our study.

In the psychophysical experiment, we found a significant reduction of mild pain perception after cathodal tDCS when the contralateral hand was stimulated with laser, which seems to contradict to findings of studies where the facilitatory anodal tDCS applied over the motor cortex successfully alleviated pain in chronic pain symptoms. One possible explanation for this discrepancy is that we examined experimentally induced acute pain in healthy subjects, whereas in previous studies the motor cortex was stimulated in chronic pain syndromes. Such chronic pathological states are characterized by both functional (i.e. reorganization of synaptic transmission) and structural alterations in cortical and subcortical areas, probably also leading to excitability changes. Another important issue is that in the first clinical studies, antinociceptive effects were reached with 20 minute-long daily sessions of $2 \mathrm{~mA}$ strong anodal tDCS; that is with twice as long and strong stimulation parameters, as the one applied in our study. Interestingly enough, even by using this protocol they did not observe significant changes immediately after tDCS, but only the following day, indicating that the effect of tDCS developed much slower than one would expect it in any other modality among healthy subjects.

The effect of anodal stimulation on warm thresholds was to some extent opposite to that of cathodal tDCS. Anodal tDCS facilitated warm sensation without influencing pain sensation or LEP amplitudes. Regarding the differential effect of anodal tDCS on warm and pain sensation, there is some evidence that certain brain regions are differentially involved into processing of warm and painful stimuli. In a functional magnetic resonance imaging study, different blood 
oxygen level-dependent signal change in the contralateral operculoinsular region was reported for painful and warm stimulation. We might speculate that the modulation of neural excitability in this region contribute to the observed shift of warm threshold and manifest in thermal hyperesthesia.

In conclusion, we have demonstrated that both cTBS and tDCS reduce subjective pain intensity and attenuate late LEP components when applied over the motor cortex. Although we found differences between cTBS and tDCS regarding the intensity of pain that was modulated, the laterality of effects and the manner to which the N2 and P2 components were changed, both stimulation techniques exhibited significant effects when compared to sham conditions. Our better understanding of the molecular effects of rTMS paradigms and tDCS is essential to further improve such antinociceptive effects. In fact, since the publication of our studies, it has been shown in the same laboratory that the D2-receptor agonist pergolide prolonged the antinociceptive and N2 amplitude reducing effects of cathodal tDCS when applied over the M1. These are very promising findings, which hopefully will facilitate the development of novel techniques for alleviating chronic pain. 


\section{Papers the thesis is based on:}

I. Poreisz C, Csifcsák G, Antal A, Levold M, Hillers F, Paulus W. Theta burst stimulation of the motor cortex reduces laser-evoked pain perception. Neuroreport. 2008; 19:193-196. Impact factor: 1.904

II. Csifcsák G, Antal A, Hillers F, Levold M, Bachmann CG, Happe S, Nitsche MA, Ellrich J, Paulus W. Modulatory effects of transcranial direct current stimulation on laser-evoked potentials. Pain Med. 2009; 10:122-132. Impact factor: 2.393

III. Csifcsák G, Nitsche MA, Baumgärtner U, Paulus W, Treede RD, Antal A. Electrophysiological correlates of reduced pain perception after theta-burst stimulation. Neuroreport. 2009; 20:1051-1055. Impact factor: 1.805

IV. Csifcsák G, Antal A. Non-invasive brain stimulation for relieving acute and chronic pain. Ideggy Szle. Accepted for publication.

\section{Cumulative impact factor: $\mathbf{6 . 1 0 2}$}

\section{Papers related to the thesis:}

I. Poreisz C, Antal A, Boros K, Brepohl N, Csifcsák G, Paulus W. Attenuation of N2 amplitude of laser-evoked potentials by theta burst stimulation of primary somatosensory cortex. Exp Brain Res. 2008; 185:611-621. Impact factor: 2.195

II. Antal A, Brepohl N, Poreisz C, Boros K, Csifcsák G, Paulus W. Transcranial direct current stimulation over somatosensory cortex decreases experimentally induced acute pain perception. Clin J Pain. 2008; 24:56-63. Impact factor: 2.889

\section{Abstracts related to the thesis:}

I. Poreisz C, Antal A, Brepohl N, Boros K, Csifcsák G, Paulus W. Transcranial direct current stimulation over somatosensory cortex decreases experimentally induced pain perception. Seventh Göttingen Meeting of the German Neuroscience Society. Göttingen, Germany, March 29 - April 1, 2007.

II. Csifcsák G, Antal A, Paulus W. Modulatory effects of transcranial direct current stimulation on acute pain perception. 6th Congress of the Federation of European Psychophysiology Societies. Budapest, Hungary, May 31 - June 3, 2006. 


\section{Acknowledgements}

I would like to express my sincere gratitude to my supervisors Professor Andrea Antal and Professor Zoltán Janka for their support throughout the years of my scientific work. Their vast knowledge and attitude towards neuroscience have been extremely valuable for me.

I would like to thank Professor Walter Paulus for the facilities and help he provided during my work at the Department of Clinical Neurophysiology in Göttingen.

I would like to give special thanks to Dr. István Szendi for his support in learning electroencephalography and for his very valuable advices and encouragement.

Among my colleagues I would like to especially thank Dr. Péter Álmos, Dr. Klára Boros, Dr. Csongor Cimmer, Dr. Szatmár Horváth, Dr. Zoltán Ambrus Kovács, Dr. Anita Must, Dr. Csaba Poreisz, Dr. György Szekeres and Dr. Márta Zimmer for their help and for creating such a friendly atmosphere both in Szeged and Göttingen.

Last but not least, I would like to express my heartfelt gratitude to my family and friends for their encouragement and endless patience. 